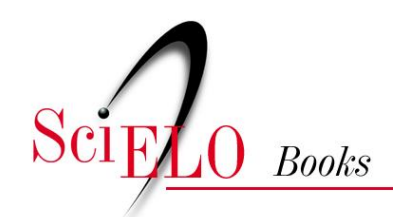

\title{
Hacia una "traducción" intercultural en las sociedades postseculares
}

\author{
Julián García Labrador
}

\section{SciELO Books / SciELO Livros / SciELO Libros}

GARCÍA LABRADOR, J. Hacia una "traducción” intercultural en las sociedades postseculares. In: GARCÉS, F., and BRAVO, R., eds. Interculturalidad. Problemáticas y perspectivas diversas [online]. Quito: Editorial Abya-Yala, 2019, pp. 13-46. ISBN: 978-9978-10-497-2. https://doi.org/10.7476/9789978104972.0002.

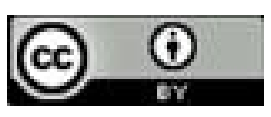

All the contents of this work, except where otherwise noted, is licensed under a Creative Commons Attribution 4.0 $\underline{\text { International license. }}$

Todo o conteúdo deste trabalho, exceto quando houver ressalva, é publicado sob a licença Creative Commons Atribição 4.0.

Todo el contenido de esta obra, excepto donde se indique lo contrario, está bajo licencia de la licencia Creative Commons Reconocimento 4.0. 


\title{
Hacia una "traducción" intercultural en las sociedades postseculares
}

\author{
Julián García Labrador \\ Universidad Politécnica Salesiana
}

El presente capítulo aborda la traducción intercultural como ejercicio epistemológico y político en las sociedades postseculares. Para ello se describe la sociedad postsecular en el pensamiento de Habermas y se critica la unilateralidad de su postura: la razón secularizada no puede ser el término último de referencia al que asimilar el resto de tradiciones culturales. Preferimos, por ello, ampliar el campo de la traducción, entendiendo que la interculturalidad opera como un horizonte que corrige y apuntala los esfuerzos por integrar el pluralismo epistémico en el proyecto político de nuestras sociedades.

\section{Interculturalidad y sociedad postsecular}

Habermas (2001a) llama "sociedad postsecular" a la nueva conciencia pública que se da en las sociedades industrializadas en torno a la relación entre modernidad y religión. Para el pensador alemán la secularización de la razón no puede obviar la riqueza semántica de las tradiciones religiosas. Si en los primeros compases de la secularización la razón tuvo que prescindir de la religión para alcanzar su carácter autónomo, ahora no puede relegar sin más los aportes derivados de las religiones.

Sorprende el hecho de que un autor tan poco dado a la confesionalidad como Jürgen Habermas reconozca el carácter positivo de la religión en el ámbito público. En efecto, se ha dado un cambio en su pensamiento, ya que, aunque el autor no profese religión alguna y siga siendo un adalid de la racionalidad crítica, asume que existe una reserva 
de sentido en las tradiciones religiosas. Las intuiciones normativas de las religiones pueden contribuir al enriquecimiento de la razón secularizada en la medida en que puedan ser traducidas a un lenguaje universal.

\section{Entre naturalismo y postmodernismo}

Cuando Habermas presenta el programa crítico de la razón secularizada se da cuenta de que el éxito de la empresa puede fracasar. La razón por si sola se expone al "descarrilamiento" del proyecto emancipatorio. Es lo que Habermas reconoce al considerar la "debilidad moral de la razón" (2007). En este sentido, acepta que las tradiciones religiosas pueden prestar un servicio de ayuda a la razón para que ésta no desfallezca en su proyecto.

El postmodernismo y el naturalismo son dos de los síntomas que indican que el proyecto moderno de secularización de la razón puede malograrse. El postmodernismo no es sino la expresión máxima de que la razón moderna habría fracasado en su proyecto emancipatorio. El debate, agudizado en las décadas de los ochenta y noventa del siglo XX, presentaba una modernidad en lucha consigo misma. La tensión entre los éxitos y fracasos de la modernidad derivaba de la misma razón. Lyotard (1987) vinculaba la condición postmoderna con la tradición europea. Para el autor francés ya no era posible pensar en los grandes relatos. La gran tradición metafísica occidental había desembocado en el reconocimiento de su caída. No era posible pensar más allá del fragmento. El pensamiento, tras la modernidad, era, así, incapaz de generar estructuras sistemáticas fuertes. Se presentaba como un "pensamiento débil" y "nihilista" (Vattimo, 1983, 1985).

Dos son las fuentes del pensamiento postmoderno: por una parte, la reflexión de la tradición ontológica destructiva de Nietzsche-Heidegger (no en vano son el binomio de referencia para Vattimo) y, por otra, la confrontación de la Teoría Crítica con el Holocausto. Cuando en 1944 Adorno y Horkheimer (2007) denunciaron que las raíces del Holocausto podían encontrarse en la racionalidad alumbrada por la Ilustración no hacían más que sentar las bases para rechazar el proyecto 
moderno de la autonomía de la razón. En tal sentido ha profundizado recientemente Zygmut Bauman (2010).

Presentar el análisis de la racionalidad moderna como "postmodernismo" implica reconocer que el relativismo que va aparejado con la crítica a la modernidad puede convertirse en metodología ideológica. No en vano el proyecto postmoderno encuentra cabida en la crítica a la modernidad europea llevada a cabo por los estudios postcoloniales. Así, la obra clásica de Said, Orientalismo (1978), se nutre de las posiciones deconstructivas de Derrida y la teoría del poder de Foucault. A partir de esta obra el programa deconstructivo del paradigma postcolonial utiliza frecuentemente la crítica al sujeto, el desmontaje del Estado moderno y el derrumbe del proyecto de la modernidad para justificar su proceder metodológico. Tanto es así que, incluso al interior de los estudios postcoloniales aparecen voces que reclaman una clarificación respecto al sentido de la crítica a la modernidad (Chakrabarty, 2011).

El naturalismo, por otra parte, ha venido a subsanar el vacío proclamado por el postmodernismo. Asumiendo la superioridad de la racionalidad científico-técnica, propugna una "objetivación cientificista de la condición humana” (Rodríguez Duplá, 2017, p. 32). La ciencia se eleva por encima de cualquier otro modo de conocimiento proclamando su superioridad técnica y práctica. Pero ello no hace sino desvelar la aporía inconclusa que denuncia la dimensión valorativa de las ciencias (Gómez, 2014). Es decir, considerando su superioridad, la ciencia se arroga para sí misma la consideración valorativa del mundo, del ser humano y de la sociedad, bajo una pretendida capa de objetividad.

Los problemas de ambas posturas aparecen cuando la sociedad pretende instaurar ciertas bases sobre las que pueda existir una convivencia real. El problema fundamental del postmodernismo es el relativismo inherente. Es decir, si el debate se mantuviera en el ámbito teórico no tendría mayor relevancia social. El hecho de que la razón pueda o no reconocerse autónomamente a sí misma no deja de ser un debate teorético. Ahora bien, dado que las instituciones sociales, jurídicas y políticas han venido dictadas por el uso práctico de esa razón, reconocer sus limitaciones implica asumir la debilidad de los actuales marcos de referencia dados para la convivencia humana. Por otro lado, 
la exaltación de la racionalidad científica como marco único de sentido convierte a la ciencia moderna en el tribunal supremo al que la sociedad puede acudir. Sin embargo, tal auto-referencialidad científica no hace sino volver una y otra vez sobre la pregunta ¿todo lo científicamente posible es socialmente deseable? Es este sentido, la trifulca entre Sloterdijk (1999) y Habermas (2001b) a propósito de la eugenesia nos lleva a reconsiderar la capacidad normativa de la agenda científica.

La crítica del postmodernismo nos pone en guardia ante la destrucción indiscriminada de los programas "post" (incluido el planteamiento post-estructural y de-colonial), remitiéndonos a la pertinencia y actualidad de la prohibición de Hume (1984) al respecto: la imposibilidad de deducir proposiciones normativas desde el reconocimiento de los hechos. A saber, de un "ser" no puede deducirse lógicamente un "deber ser". Incluso Kant, al aceptar la crítica del mismo Hume, se vio obligado a reconocer el uso trascendental de la razón ante la ausencia de referencias. Una vez que Kant había destruido las posibilidades científicas de la metafísica, reconoció que la razón, vacía de conocimiento, podía seguir funcionando como ideal regulativo. La crítica del absolutismo naturalista nos coloca ante la necesidad de calibrar mejor las relaciones entre ciencia y política, toda vez que las implicaciones prácticas de la ciencia son cada vez más notorias y que los presupuestos de investigación dependen de la priorización política.

Esto nos lleva a las puertas de los dos sentidos que podemos descubrir en el concepto de Habermas a propósito de la sociedad postsecular: el sentido descriptivo y el sentido normativo.

\section{Los usos descriptivo y normativo de la sociedad postsecular}

Rodríguez Duplá (2017) ha desvelado los entresijos de los dos sentidos del concepto habermasiano de sociedad postsecular: descriptivo y normativo. El sentido descriptivo pretende mostrar el resultado de la separación entre razón y tradiciones religiosas en la época moderna. El sentido normativo intenta traducir las intuiciones morales de las tradiciones religiosas a un lenguaje universal para que la razón moderna pueda enriquecerse y fortalecerse; en un sentido normativo, las tradicio- 
nes religiosas aportan sus contenidos morales al programa emancipatorio de la razón moderna.

El sentido descriptivo de la sociedad postsecular parte de la realización del proceso de secularización en Europa en la época moderna, según el cual la religión se separa de la razón en el ámbito público. Una sociedad secularizada es entonces aquella en la que las decisiones sobre los asuntos públicos se realizan sobre la base de una racionalidad independiente y compartida por los ciudadanos. En mi opinión, Habermas modela su concepto descriptivo de sociedad postsecular desde la presunción de un proceso puro e incontaminado, cuando, en realidad, el desarrollo de la secularización y sus transformaciones son mucho más complejas. Así, las posturas en torno a la secularización van desde aquellos que presentan una colisión entre el régimen de cristiandad católica y las nuevas estructuras científicas, capitalistas y políticas de la modernidad (Martin, 1978) hasta aquellos que descubren la contribución del cristianismo a la filosofía política de la época moderna (Gauchet, 2005). Para Talal Asad (2003) en el desarrollo de la secularización se producen inversiones e influencias mutuas entre lo secular y la religión cristiana. Charles Taylor (2007) define la época moderna como una época secular (Secular Age), cuya dinámica interna consiste en la referencia a un marco inmanente. Una de las mejores descripciones analíticas de la secularización la encontramos en José Casanova:

Como conceptualización analítica de un proceso histórico, la secularización es una categoría que tiene sentido dentro del contexto de las dinámicas internas y externas particulares de la transformación del cristianismo europeo occidental desde la Edad Media hasta el presente. (2006, p. 12)

El problema derivado de presentar una descripción analítica de la secularización implica reconocer que encontrar una sociedad postsecular tan prístina como la que presenta Habermas es tremendamente difícil, por no decir imposible. Es más, profundizando en el concepto analítico Casanova (1994) distingue tres aspectos de la secularización: el declinar de las creencias y las prácticas religiosas, la privatización de la religión y la independencia de la esfera secular respecto a la religión. 
Sin embargo, descarta que sea un fenómeno universal. No hay una regla global — "universal rule" - (Casanova, 2006, p. 17) que permita hablar de una secularización total, incluso en un mundo globalizado.

En sentido descriptivo, una sociedad postsecular nunca se ha dado y es posible que nunca exista. Además, el propósito de describir una sociedad según la base de la relación histórica con las instancias de una religión es incompleto. Es posible que al interior de una sociedad determinada no haya uno, sino varios procesos de secularización en diferentes fases de desarrollo; e - incluso - pueden darse procesos inversos a la secularización prevista por Habermas, como indica Hans Joas (2002) respecto a la realidad de los Estados Unidos. Por ello, este autor desaconseja encarecidamente el uso sociológico del concepto "sociedad postsecular".

A la crítica de los teóricos de la secularización hemos de añadir el carácter polifónico y multicultural de las sociedades actuales. El discurso habermasiano descansa sobre la consideración de la sociedad moderna europea y sus relaciones entre lo secular y la religión cristiana. Las sociedades actuales y los problemas de lo secular con la religión ni se ciñen a una cultura única ni al diálogo con una única tradición religiosa. Que son sociedades multiculturales es un hecho, cuyo reconocimiento cuenta con un breve recorrido. A pesar de cierto estatismo cultural, la postura de Kymlicka (1996) reveló en los noventa el problema fundamental que Habermas había pasado por alto: el multiculturalismo. No podemos hablar, pues, de sociedad postsecular sino más bien de sociedades postseculares y ello en un sentido no descriptivo. El hecho de que Kymlicka se refiera a la sociedad canadiense como una sociedad multicultural corrige la tendencia a pensar las sociedades occidentales actuales como la decantación de una cultura única. Y esta prevención influye de manera decisiva en la consideración de la secularización, puesto que la asociación de cultura y tradición religiosa es un hecho más que frecuente.

Así pues, si el carácter descriptivo de la sociedad postsecular presenta problemas difícilmente subsanables, tal vez el uso normativo del mismo pueda hacer pensar en ciertas vías de aplicación. Tal es el camino emprendido por Habermas. El uso normativo del concepto sociedad postsecular incide en la actitud hacia la religión y en la confianza depositada en la razón secular. Habermas espera que el proyecto moderno 
pueda llegar a su máxima expresión en el uso de la racionalidad crítica y emancipada. Confía en que el proyecto pueda llevarse a cabo. Pero también sospecha que pueda echarse a perder. Si la razón secular, azotada por el postmodernismo y la autosuficiencia del naturalismo, no es capaz de generar contenidos normativos significativos para la sociedad, fracasará. La razón moderna, "dejada a su dinámica inmanente, puede terminar destruyendo sus más preciosas conquistas" (Rodríguez Duplá, 2017, p. 30). Por ello, Habermas plantea la secularización en los términos de un proceso de aprendizaje mutuo entre la razón moderna y las tradiciones religiosas, de tal manera que las intuiciones morales de las religiones puedan ser traducidas a un lenguaje universal y, de esta manera, contribuir al despliegue de las virtualidades de la razón. De esta manera, Habermas trata de salir al paso de la tensión entre postmodernismo y naturalismo, así como de las limitaciones de las teorías de la justicia que, nacidas al albur de la razón moderna, no pueden dar cuenta de la dimensión solidaria y comunitaria de la justicia:

Es precisamente este "déficit político" de las éticas de la justicia el que lleva a Habermas a volver la vista a las grandes tradiciones religiosas, en las que la solidaridad se alimenta de la conciencia de compartir un destino salvífico común, que en último término abraza a toda la humanidad. (Rodríguez Duplá, 2017, p. 31)

Así, la razón no puede sin más "sustituir ni desalojar a la religión" (Habermas, 1988, p. 60). No obstante, la consideración positiva de Habermas acerca de las religiones tiene un sentido coadyuvante. Es posible que, deudor de la visión weberiana de las relaciones entre modernidad y religión, mantenga una postura utilitarista. Es más, en mi opinión, este "estrechamiento funcionalista y evolucionista" (Mardones, 1998, p. 77) responde a un esquema teleológico que recorre el pensamiento moderno y ante el que sucumben no pocos autores.

Más allá del papel de las religiones, el sentido normativo de la sociedad postsecular nos enfrenta con algunas cuestiones que la postura postmetafísica de Habermas (1988) no puede solucionar. ¿Por qué habría de ser la razón secularizada el instrumento privilegiado para establecer la convivencia en nuestras sociedades multiculturales? 
El sentido dialógico de la secularización (enriquecimiento mutuo entre razón secular y tradiciones religiosas) nos conduce a valorar el diálogo al interior de las sociedades multiculturales como una poderosa herramienta de convivencia. Pero al mismo tiempo que lo valoramos, la experiencia cotidiana nos demanda una concreción del programa habermasiano y una revisión del concepto de diálogo "dialógico".

Considero que su concepto de sociedad postsecular puede enriquecerse en una doble perspectiva. Por un lado, teniendo en cuenta el pluralismo y la globalización de las sociedades contemporáneas conviene hablar de sociedades postseculares en un sentido no metafísico, es decir, en un sentido que tenga en cuenta la mixtura de los proceso económicos, sociales y científicos de las sociedades contemporáneas y no sólo desde la relación entre la razón (entendida como facultad humana que permite una autonomía discursiva en el uso público) y la fe (como doctrina normativa de la religión cristiana en Europa). Cuando Habermas adopta una postura postmetafísica hace gala de un eurocentrismo filosófico difícilmente compatible con los presupuestos filosóficos de otras tradiciones culturales en las que, o bien no se ha producido el proceso de secularización, o bien se ha producido en una dirección diferente a la europea. Por otro lado, el sentido normativo del concepto "sociedad secular" se ve acompañado por el sentido normativo del programa multicultural: "el multiculturalismo es un programa normativo que afirma el valor positivo de las culturas o de la diversidad cultural, y que aspira a elaborar los mecanismos jurídicos que las protejan, fomenten y hagan perdurar en el tiempo" (Loewe, 2012, p. 48).

Teniendo en cuenta ambas perspectivas entendemos que, si el déficit político de las éticas de la justicia hacía que Habermas volviera la vista a las tradiciones religiosas, no por ello quedaba solucionado el asunto. Precisamente ese déficit político ha motivado un acalorado debate en torno a la problemática jurídica de las sociedades multiculturales. El debate se ha centrado en cómo generar un espacio jurídico suficiente para que todas las culturas puedan desarrollar de manera plena sus expresiones y prácticas culturales. Habermas no iba tan lejos. Tan sólo quería apropiarse críticamente de las intuiciones morales de las tradiciones religiosas y traducirlas a un lenguaje universal. No obstante, el 
problema de fondo es el mismo: ¿cómo discriminar unas intuiciones de otras? o, en el lenguaje normativo del proyecto multicultural ¿cómo dar cauce a las demandas jurídicas de una determinada cultura - "ciudadanía diferenciada" (Young, 1990) — sin vulnerar los derechos de otras?

El hecho de implementar la "ciudadanía multicultural" (Kymlicka, 1996) en las sociedades postseculares nos lleva a plantearnos cómo entender lo multicultural en su dimensión sistemática. En este sentido, creo que el sentido normativo de las sociedades postseculares va de la mano del programa normativo de la multiculturalidad.

\section{Interculturalidad como agenda política}

Las políticas multiculturales son variadas y no siempre coincidentes. En muchas ocasiones, su implementación choca con la pretendida igualdad del Estado de derecho. Es decir, teniendo en cuenta que la ciudadanía se sustenta sobre el concepto de igualdad, el hecho de hablar de derechos culturales diferenciados vendría a reparar la situación de injusticia histórica de los grupos minoritarios. Sin embargo, considerar la utopía multicultural desde la perspectiva de los contenidos proposicionales de las diferentes culturas nos puede llevar a la imposibilidad lógica de crear espacios sociales y jurídicos para cada particularidad cultural. Como indica Barry "el inevitable problema es que culturas tienen un contenido proposicional” (2001, p. 270) y esto deriva en la aporía valorativa de las culturas en un contexto social. Si reconocemos con Taylor (1994) que el problema del reconocimiento cultural deriva en la autorreferencialidad de los individuos, estamos convirtiendo la pluralidad cultural en un asunto de máxima trascendencia. Pero al mismo tiempo no sabemos cómo se "valora" culturalmente la diferencia al interior de las sociedades. ¿Puede una cultura ser más valorada que otra?

Ante este problema podríamos asumir con Tully (1995) la pertinencia de un relativismo cultural, incluso en su carácter normativo. Quiere esto decir que cada universo cultural es también un conjunto autorreferenciado independiente. La normatividad de cada universo cultural es ajena al resto. Es esta una postura extrema que imposibilita la coexistencia en un marco jurídico común en el seno de una sociedad 
y que, además, no ofrece vías de solución en caso de colisión normativa de las tradiciones culturales.

Posturas menos extremistas tratan de conjugar la diversidad cultural con la igualdad inherente en derechos y oportunidades. En este sentido, Parekh (2006) ha propuesto un reconocimiento culturalmente sensible de la igualdad. Parekh tiene el mérito de haber intentado conciliar el planteamiento igualitario de los derechos civiles con el reconocimiento de la diversidad cultural. No obstante, no soluciona el problema de base como es la existencia de prácticas culturales que pueden colisionar con los derechos fundamentales. La neutralidad cultural no implica aceptar, por ejemplo, la mutilación genital femenina. Además, el reconocimiento de la diversidad cultural no puede amparase en una libertad de conciencia que se superponga a los mínimos de convivencia al interior de las sociedades.

Los problemas del multiculturalismo en relación con el liberalismo político se convierten en auténticas aporías que, desde mi punto de vista, parten de una noción estanca de la cultura y de una consideración unidimensional de la identidad. Entender la cultura como un cajón estanco, inmóvil no se corresponde con la realidad diaria de muchos individuos. Es lo que ha reconocido Waldmüller: "buena parte de los individuos en realidad registran al mismo tiempo múltiples pertenencias culturales tanto en el mundo real como en el simulado" (2017, p. 79). Asumiendo la cultura como un entramado dinámico y teniendo en cuenta las pertenencias múltiples, la cuestión del carácter normativo del multiculturalismo toma una dirección diferente. Partiendo, además, de que nuestra humanidad se juega en el ámbito de las identidades compartidas (Nussbaum, 1997), la cuestión política se revela como una acción a realizar desde la interculturalidad.

Distinguimos así, la multiculturalidad, que considera la cultura como un depósito estanco de contenidos y prácticas y la interculturalidad, que asume el carácter dinámico y transformador de las culturas. Asumimos las identidades compartidas como un dato innegable de nuestras sociedades y destacamos que su construcción es factible dentro de una agenda política intercultural. Cuando los autores se refieren, por ejemplo, al caso de Ecuador, apuntan en esta misma dirección. Altmann revela que, 
sin el carácter normativo, la interculturalidad queda como un concepto vacío y, lo que es peor, susceptible de ser instrumentalizado. Por ello, propone la interculturalidad como concepto político o normativo, el cual puede ser pensado desde dos orillas: "desde los excluidos y marginados que demandan la interculturalidad como la posibilidad de participar sin renegar su identidad; y desde los estados" (Altmann, 2017, p. 15). En el primer caso la demanda intercultural encaja con el concepto político de la plurinacionalidad (Altmann, 2013), entendido como instrumento de reconocimiento de autonomía administrativa y cierta autogestión territorial al interior de los estados. En el segundo caso, nos encontramos con el trato dispensado a los agentes "otros" entendidos como no partícipes de la conformación nacional. Es decir, el uso político de la interculturalidad desde los estados hace que las poblaciones tradicionalmente excluidas difícilmente puedan participar en los proyectos políticos nacionales, dando al traste con el pleno desarrollo de la plurinacionalidad.

Pienso que los estados no han entendido aún que la agenda política intercultural puede contribuir tanto al fortalecimiento del Estado como a la construcción de las identidades plurales de sus ciudadanos. Es más, una agenda política intercultural nos permite organizar nuestras sociedades postseculares llevando la propuesta de Habermas más allá del sometimiento de contenidos culturales y religiosos a la razón moderna. En 1964 Searle propuso una solución al problema metaético de Hume (cómo derivar enunciados normativos de enunciados descriptivos) desde la filosofía analítica. Para Searle en su teoría de los Actos de habla, las promesas son enunciados que implican un compromiso respecto al futuro. Las promesas son actos ilocutivos, los cuales contemplan las condiciones empíricas para su realización. Dicho de otro modo, la distinción entre los enunciados descriptivos y los evaluativos no tendría razón de ser: "hemos mostrado que "promesa" es una palabra evaluativa, pero como también es puramente descriptiva, realmente hemos mostrado que toda la distinción debe ser reexaminada." (Searle, 1964, p. 58).

Años más tarde, Searle reevaluó su propia propuesta incorporando la pragmática lingüística. En este sentido, entendió que el lenguaje es una institución social que nos permite construir la realidad (Searle, 1997). La significatividad de los enunciados se logra en el uso de la red lingüística 
socialmente compartida. De esta manera, la significatividad de las promesas se da en el conjunto de reglas constitutivas del lenguaje. Lo que hace Searle es lo siguiente: contemplar la institución de la promesa en su contexto lingüístico para derivar la auto-obligación de cumplirla.

Con ello, Searle abre el camino para las relaciones entre moral y lenguaje, teniendo en cuenta que, aunque los enunciados de hecho nos permitan conocer la realidad, estos mismos enunciados han de ser evaluados. Esto es, la institución moral agrupa los dos tipos de enunciados borrando la férrea distinción del ala más extrema de la filosofía analítica.

La propuesta de Searle nos permite ampliar el marco de lo que Barry establecía como "contenidos proposicionales" de la cultura. De esta manera, para entender cualquier contenido cultural conviene aproximarse lingüísticamente al entramado de relaciones sociales del que parte. Lo mismo que para Searle era importante el conjunto de condiciones empíricas que la lengua inglesa suponía para el cumplimiento de una promesa, los contenidos proposicionales de cualquier cultura no pueden entenderse sin tener en cuenta su lenguaje cultural como institución social.

Con ello, llegamos al punto en el cual Habermas, la interculturalidad y la filosofía intercultural entran en relación. Habermas proponía un trasvase de los contenidos de las tradiciones religiosas a la razón moderna. La interculturalidad como agenda política permite entender las culturas e identidades de forma dinámica y permeable. Tanto la perspectiva de Habermas como la de la agenda intercultural conllevan la asunción del carácter normativo de su implementación, bien como una traducción hacia la razón moderna en el caso de Habermas, bien como la satisfacción de ciertas demandas culturales en el caso de la agenda política intercultural. Searle nos ha hecho ver cómo los contenidos descriptivos y los normativos no están, en realidad, tan separados. El hecho de que la construcción de la moral implique para Searle la reunión de enunciados descriptivos y valorativos nos hace pensar que es posible la construcción de una moral intercultural en nuestras sociedades postseculares. Aquí se hace plausible la necesidad de un concepto normativo no coercitivo. Éste lo encontramos en el paradigma de "horizonte", con el que Fornet-Betancourt (2004) caracteriza la tarea de una filosofía au- 
ténticamente intercultural. El concepto de horizonte es, en mi opinión, uno de los aspectos más relevantes y liberadores de su filosofía, ya que ofrece la posibilidad de comprender de manera no coercitiva la tarea intercultural y de facilitar un diálogo intercultural sin anclajes previos.

A continuación se desarrolla el argumento en dos movimientos. En primer lugar, propongo una crítica al logocentrismo de la propuesta de Habermas. Después planteo una salida al mismo entendiendo que la traducción intercultural es la tarea más apropiada para la construcción de una moral intercultural en nuestras sociedades postseculares.

\section{Logocentrismo}

\section{Las tres etapas de Habermas}

Distinguimos en Habermas tres momentos en lo que respecta al proceso de secularización de la razón moderna. En primera instancia, siguiendo la pista de Max Weber, Habermas se muestra pesimista respecto a la relevancia de la religión en la sociedad secularizada. En 1981 publicaba Teoría de la acción comunicativa donde se hacía eco del desencantamiento weberiano del mundo, indicando que aquella imagen unificada del mundo, propia de la época de cristiandad, se estaba desmoronando. En tal situación, la religión no tenía otro destino que desaparecer. La autoridad de lo santo había quedado sustituida por la autoridad del consenso racional y no quedaba sino esperar que la religión quede conminada al ámbito privado. En un segundo momento, Habermas asume que la religión no podrá ser desalojada de las sociedades secularizadas, aunque para poder expresar de manera válida sus intuiciones ha de traducirlas al lenguaje racional, propio de la modernidad. En tal sentido, considera que, en una sociedad pluralista, las disputas entre religiones sólo podrían ser resueltas si las comunidades religiosas inician un proceso de reflexión racional asumiendo los éxitos de la modernidad. En un tercer momento, a partir de 2001, Habermas reconoce la debilidad de la razón moderna y la posibilidad de que fracase en su proyecto emancipatorio. Es en este sentido en el que asume la necesidad de contar con las intuiciones morales de las tradiciones religiosas, para que la razón moderna no fracase en sus proyectos. 
Las tres etapas del pensamiento de Habermas en torno a la secularización tienen un denominador común: la centralidad de la razón. Aunque corrija el inicial rechazo de la religión y admita la presencia de las comunidades religiosas en las sociedades pluralistas, el término de referencia al cual han de asimilarse es la razón moderna. Es más, Habermas asume que la traducción de los contenidos religiosos a la razón moderna conduce a la aceptación de los mismos, dado su carácter universal. Es decir, Habermas equipara la razón moderna con el lenguaje universal, reduciendo, en gran medida, la razón a una dimensión discursiva.

La posición logocéntrica de Habermas es más que evidente. De Klages (1971) a Derrida (1986) el logocentrismo ha sido descrito como la tendencia del pensamiento occidental a situar el logos como el eje sobre el que bascula todo el armazón especulativo. Verdad, lógica, mundo y razón son asumibles en virtud de su conversión al logos. Desde Platón hasta nuestros días, la razón se ha caracterizado por esta centralidad, la cual parte del supuesto origen común de todo conocimiento. Para Derrida el logocentrismo impide el contacto con el pensamiento puro ya que siempre estaremos anhelando un centro que de sentido (impidiendo reconocer la diferencia) y por ello indica que la metafísica está contaminada.

A pesar de su anti-intelectualismo romántico, la postura de Klages permite entender que el logocentrismo está presente en no pocos autores. El caso de Habermas es paradigmático. No sólo pone la razón como la instancia última que puede dirimir las diferencias entre credos religiosos, sino que asume su universalidad como justificación de la traducción de contenidos morales y religiosos. Además, el logocentrismo habermasiano queda en evidencia desde el momento en el que presenta la genealogía de la razón moderna. Habermas interpreta la época axial de Jaspers desde la preeminencia del pensamiento racional. Jaspers (1949) había indicado que entre los años 800 y 200 a. C. tiene lugar la era axial, en la que se produjo una revolución en China, India y los pueblos mediterráneos. Tal revolución se caracteriza por la conciencia que el hombre adquiere de sí mismo, la aparición de la actividad filosófico-reflexiva en público, el surgimiento de la conciencia histórica y el universalismo moral. Las religiones preaxiales se caracterizaban por el equilibrio del orden social y cósmico. Lo sagrado afectaba, de esta manera, todas las 
esferas de la vida. Sin embargo, las religiones y filosofías que surgen a partir de la época axial se centran en la salvación, la liberación y la redención. Se postulan a partir de entonces como sistemas universalistas y hacen hincapié en la responsabilidad personal.

Habermas atisba en este momento la génesis de lo que después será el pensamiento moderno. El surgimiento de la instancia reflexiva del ser humano sólo puede hacerse, para él, a expensas del pensamiento mágico. El mito ha de quedar atrás. Precisamente, las tres características que Habermas (2007) cree encontrar en la época axial son las características principales del pensamiento de la modernidad: conciencia histórica, moral universal y responsabilidad personal.

\section{La insuficiencia de los centrismos}

Franz Wimmer (2004, pp. 54-58) presenta cuatro modalidades (centrismos) en las que puede producirse el encuentro entre culturas. En primer lugar, el centrismo expansivo, según el cual la propia cultura sería superior al resto. La dinámica que corresponde al centrismo expansivo es la de la imposición cultural. En segundo lugar, el centrismo integracionista, que considera que hay verdades universalmente válidas y que las culturas son momentos diferentes de aproximación a la verdad. Este centrismo sustenta la no intervención cultural, asumiendo que, en algún momento, serán las otras culturas las que asuman esas verdades universales. En tercer lugar, el centrismo separatista considera que los puentes entre diferentes culturas están rotos. La comunicación no es posible. Las etnofilosofías — señaladas por Estermann (2012)—, el indigenismo radical y el choque de civilizaciones de Huntington (1996) encarnarían este centrismo. En cuarto y último lugar, el autor propone el centrismo tentativo, según el cual existe voluntad de encuentro y posibilidad de acercamiento entre culturas. El autor propone la figura del polílogo, el cual permite cuestionar la propia cultura y superar las supuestas diferencias ontológicas entre las diferentes culturas:

Un polílogo intercultural bajo un centrismo tentativo requiere como condición sine qua non que todos los participantes dediquen su educación, voluntad, empatía y apertura a desarrollar entre sí nuevos ho- 
rizontes verdaderamente interculturales. De ahí que la filosofía intercultural plantee propuestas metodológicas adicionales: entre otras, la descripción, traducción y reconstrucción adecuada de fenómenos culturales... (Waldmüller, 2017, p. 67)

La postura postsecular de la última etapa de Habermas corresponde con el centrismo integracionista, según el cual existen verdades universales al que las culturas llegarían en su proceso de maduración. La razón moderna sería la matriz última para depositar las intuiciones contenidas en las tradiciones culturales (llegados a este punto, vamos más allá de Habermas y hablamos no sólo de tradiciones religiosas sino de tradiciones culturales, teniendo en cuenta el contexto pluralista en que nos encontramos). Este proyecto integracionista de Habermas es, en realidad, una apuesta reduccionista, ya que la última palabra del proceso la tiene la razón moderna, tal como se ha ido gestando en la tradición occidental.

Creo que Wimmer y su polílogo puede corregir el proyecto de Habermas en una dirección auténticamente intercultural. Bien es cierto que el mismo término "polílogo" adolece de un logocentrismo larvado, asumiendo injustificadamente que las diferentes culturas se expresan en los mismos niveles y formas de racionalidad, supuesto que ha de ser sometido a un análisis más profundo. Con todo, si atendemos al texto arriba citado podemos distinguir dos aspectos relevantes para el asunto que estamos tratando.

En primer lugar, el hecho de que el centrismo tentativo no asuma una postura definida nos previene contra los dogmatismos interculturales. Por ello, se habla de "horizontes interculturales", haciendo gala de una necesaria apertura en todos los procesos de intercambio. La interculturalidad como agenda política demanda una sana libertad para que puedan darse las condiciones de las que habla Waldmüller. Para garantizarlo se obvia el término ad quem. Hablando de interculturalidad se puede presentar un horizonte $\mathrm{u}$ horizontes, pero no un dogma o una hoja de ruta impuesta, ya que estaríamos implementando cualquiera de los tres primeros centrismos. Tanto desde la filosofía de Fornet-Betancourt como desde el modelo polilógico de Wimmer, la noción de horizonte cobra carta de naturaleza en el proceso intercultural. 
En segundo lugar, quiero destacar que, entre las propuestas metodológicas, descuella especialmente la tarea de traducción. Junto a la descripción y reconstrucción de fenómenos culturales, pienso que la construcción de horizontes interculturales, políticamente relevantes, se juega en la tarea de la traducción. No pensemos sin embargo en términos estrictamente lingüísticos. No se trata de traducir un término por otro. No es una actividad especular, ni una reproducción de esquemas formales. No es tampoco una mera transferencia de contenidos culturales, sino un proceso de intercambio, transformación y diferenciación.

\section{Traducción y sistemas epistémicos}

Comencemos este apartado indicando que la traducción como mera equivalencia cultural no existe. En la traducción cultural tenemos presente que lo que está en juego es la representación del otro. Por ello, en el pasado nos encontrábamos con una traducción "aclimatada" o "extranjerizada" (Carbonell i Cortés, 1997), la cual se basaba en la manipulación de esa representación. En mi opinión, la traducción cultural siempre va a habérselas con lo otro, lo cual conlleva tener en cuenta un par de observaciones:

En primer lugar, en cualquier traducción cultural hemos de contar con lo "intraducible". Es decir, el juego entre signo y significado de una cultura dada puede no tener equivalencia ni contexto en la cultura de destino. Por ello, la traducción cultural no puede obviar los contextos de producción significativa. Así, el proceso de traducción ha de involucrar todo el abanico epistémico posible: "de arriba abajo", del "macro-texto al micro-texto" "del texto al signo" (Carbonell i Cortés, 1997, p. 115). Con cada ejercicio de traducción asistimos a una presentación de las visiones del mundo, de posiciones filosóficas y de valores compartidos, los cuales no sólo se implementan en textos sino también en prácticas culturales:

No es adecuado limitarse a tomar conciencia de los sistemas semióticos que producen los signos de la cultura y su diseminación. Mucho más importante es que estamos enfrentados al desafío de leer, en el presente de una performance cultural específica, las huellas de todos esos diversos discursos e instituciones disciplinarios de saber que constituyen la condición y los contextos de la cultura. (Bhabha, 1994, p. 199) 
En segundo lugar, la traducción cultural pone en marcha la dinámica de la diferencia. Como indica Bhabha, la diferencia cultural "introduce en el proceso del juicio y la interpretación cultural ese repentino estremecimiento del tiempo sucesivo, no sincrónico, de la significación, o la interrupción de la pregunta suplementaría que he elaborado antes" (1994, p. 199). Quiere esto decir que la diferencia cultural, lejos de ser suprimida por la traducción cultural, entra de lleno en la cuestión. Bhabha introduce el concepto de "desfase" (lagging) cultural para indicar la "estructura misma de la diferencia" (1994, p. 297). Dicho desfase no puede, empero, ser negado, sino más bien "negociado": "lo que parece lo "mismo" dentro de las culturas sea negociado en el desfase temporal del "signo" que constituye el campo intersubjetiva social" (1994, p. 297). El hecho de negociar el desfase implica tener en cuenta la dinámica de la diferencia en el ejercicio de traducción. Es esta la razón por la que Derrida tachaba el pensamiento occidental como logocéntrico: no permitía reconocer la diferencia.

Teniendo en cuenta estas dos observaciones entendemos que la traducción cultural se convierte en un ejercicio de construcción de identidades y esto tiene consecuencias políticas de primer orden. "Las designaciones de diferencia cultural interpelan formas de identidad que, en razón de su continua implicación en otros sistemas simbólicos, son siempre "incompletas" o abiertas a la traducción cultural” (Bhabha, 1994, p. 199). Es decir, el ejercicio de traducción se desarrolla en torno a las identidades compartidas de Nussbaum. De esta manera, el reconocimiento de las identidades compartidas y de la diferencia cultural genera nuevos espacios interculturales en virtud del ejercicio de traducción. Es lo que Homi Bhabha llama sitios híbridos:

En el impulso incansable de la traducción cultural, los sitios híbridos de sentido abren una hendidura en el lenguaje de la cultura que sugiere que la similitud del símbolo, tal como juega a través de los sitios culturales, no debe oscurecer el hecho de que la repetición del signo es, en cada práctica social específica, a la vez diferente y diferencial. (1994, p. 200)

Entendiendo la traducción cultural —en este sentido- podemos encontrar en la agenda política intercultural la oportunidad de 
construcción de identidades. Por ello, la traducción intercultural opera como un espacio de fundación de ciudadanía. La agenda política intercultural en las sociedades postseculares pone contra las cuerdas la postura de Habermas. Así, la tarea no es ya traducir los contenidos de las tradiciones religiosos (culturales) a la razón moderna, sino permitir un ejercicio de traducción cultural entre los diferentes sistemas epistémicos. La traducción intercultural queda así como:

La relación entre las condiciones de la producción del conocimiento en una cultura dada y como el saber proveniente de un contexto cultural diferente que se reubica y se reinterpreta de acuerdo con las condiciones, en las cuales todo conocimiento tiene lugar. (Carbonell i Cortés, 1997, p. 48)

Entender la traducción cultural en términos de relación entre las condiciones de producción de conocimiento nos lleva a tratar una cuestión aledaña: los sistemas epistémicos. Bhabha entendía que tanto los sistemas semióticos como las huellas dejadas por estos eran objeto de traducción. Severi (2014) se refiere a los "sistemas de pensamiento". Es más Severi (2015) considera la traducción como el nuevo espacio epistemológico. Mi postura asume lo dicho por estos autores, pero añade la autovaloración referencial a dichos sistemas. Por esta razón, prefiero hablar de sistemas epistémicos, reconociendo con Searle la dificultad de deslindar los enunciados descriptivos de los valorativos.

¿Por qué hablar de sistemas epistémicos? Lloyd (2014) ha referido el caso de lo ocurrido en el encuentro China-Occidente en el siglo XVI. Los misioneros jesuitas iniciaron un diálogo de carácter científico con los sabios chinos acerca de las unidades básicas de la realidad. Los jesuitas se encontraron con que los términos chinos para "tierra", "agua", "fuego" no correspondían con la metafísica subyacente a la física escolástica en que ellos habían sido formados. Los jesuitas aún pensaban que tal física se correspondía con un sistema de conocimiento universalmente compartido. La realidad era muy distinta. Términos chinos como wu xing hacían referencia a "procesos" no a "elementos estáticos". "Entonces, donde los jesuitas - y antes que ellos el propio Aristóteles - habrían visto cosas, los chinos vieron eventos" (Lloyd, 2014, p. 23). Los jesuitas habían sido formados en un sistema epistémico donde la 
metafísica de la sustancia era valorada por encima de todo. Por ello, les fue tan difícil entender que el dinamismo y el cambio eran, en realidad, aquello a lo que se referían sus términos. La lectura tomista de Aristóteles había privilegiado una de las categorías del ser (sustancia) por encima de otras que hacían referencia al dinamismo de la realidad (relación). De esta manera, la valoración epistémica de la permanencia impedía a los jesuitas comprender que el sistema epistémico chino no había ejecutado la reducción unidimensional de la realidad tal como había procurado la escolástica occidental.

$\mathrm{Al}$ considerar los sistemas epistémicos desde esta perspectiva dejamos atrás una concepción estrecha de la racionalidad, que distingue maniqueamente entre lo racional y lo irracional. Siguiendo a Vygotsky (1978) recogemos la siguiente apreciación de Carlo Severi:

Sin ignorar los problemas planteados por las diferencias culturales, Vygotsky elaboró una concepción multifacética del ejercicio del pensamiento, que incluye no solo inferencia racional, sino también metalingüística, metacomunicacional, estética ("pensamiento a través de nuestros ojos", como lo define Joyce), y pensamiento narrativo. (2014, p. 42)

Partiendo de este presupuesto, Severi, sale al paso de las objeciones que afectan a la posible traducción cultural: la identificación lenguaje-cultura, la relación entre lenguaje y pensamiento y el binomio extremista universalismo-relativismo. Severi parte de la práctica para elaborar la teoría. Considera que no es a través de la teoría sino de la práctica etnográfica como la antropología puede apreciar el proceso de traducción en toda su extensión dinámica:

Considera la etnografía de la traducción como una oportunidad para observar la dinámica de los procesos de pensamiento y estudiar cómo operan, tanto para adaptarse a las limitaciones como para explotar las posibilidades, en diferentes contextos culturales. (Severi, 2014, p. 44)

Pensemos, por ejemplo, en el giro ontológico de la antropología. El trabajo etnográfico en torno a las sociedades amerindias ha posibilitado que la antropología llegue a cuestionar la ontología tradicional occidental. Tal ontología estaba asociada a un sistema epistémico en el 
que la univocidad del ser se había convertido el centro de referencia de todo lo demás. Cuando Viveiros de Castro (1998) plantea la necesidad de pensar la pluralidad de la cosmovisión amazónica no en términos de propiedades del ser (en línea aristotélica), sino como un agregado potencial de múltiples seres en una misma entidad, está realizando un ejercicio de traducción cultural. El perspectivismo adoptado por Viveiros de Castro no es sino un camino exploratorio en el que la dinámica del pensamiento amerindio se pone en ejercicio en un contexto diferente.

\section{Más allá del logos. El horizonte de la trasposición}

Pensar la interculturalidad como un proceso de traducción cultural es, a mi entender, una de las mejores formas de poner en marcha la agenda política. Es notorio que cuando se critica la falta de implementación de la interculturalidad se hable de ello como una "despolitización de lo intercultural" (Gómez Rendón, 2017, p. 114). Asistimos a la conversión de las prácticas culturales en espectáculo, donde la lengua es considerada un indicador de autenticidad. Se trata de un ejercicio no de traducción cultural, cuanto de exhibición del "esencialismo identitario" (Spivak, 1987) en el que los presupuestos no explícitos apuntan a una concepción estática y a-histórica de la cultura y la identidad.

Para concluir esta exploración teórica en torno a las posibilidades de la traducción intercultural en las sociedades postseculares, propongo tres vías que complementan y abren perspectivas respecto a lo dicho hasta ahora: una contextualización de la modernidad de Habermas, la constitución de la filosofía intercultural como condición trascendental de traducción cultural y una reevaluación de la traducción cultural, sus límites y sus transgresiones.

\section{Habermas y las tradiciones respecto a la modernidad}

$\mathrm{Al}$ acusar a Habermas de logocentrismo no podemos sin más apelar a la ingenuidad del autor alemán, tan comprometido, por otro lado, por un uso de la racionalidad humana que vaya más allá del mero uso instrumental. En efecto, me parece loable el intento por llevar más allá de sus limitaciones las intuiciones de la Escuela de Frankfurt. Para Ha- 
bermas la modernidad es un proyecto inacabado en el que la razón aún puede fortalecerse y asumir su tarea liberadora con carácter universal.

Resulta difícil implementar una agenda intercultural partiendo de estos presupuestos. No pretendo, sin embargo, hacer una enmienda a la totalidad. El hecho de que Habermas se postule como adalid de la modernidad encuentra su razón de ser en las conquistas que el pensador alemán asigna a la época moderna. En Habermas se unen la crítica kantiana y la filosofía de Marx. A través de la interacción entre la acción comunicativa y el mundo de la vida, Habermas pretende salir al paso de la reducción marxiana de la concepción del ser humano. En Habermas, la praxis humana va más allá del mundo trabajo. Teniendo en cuenta esta reducción de Marx, Habermas (1989) realiza una nueva lectura de Hegel que pueda sacar a la luz las dimensiones de la subjetividad que habían quedado olvidadas. Destaca cuatro dimensiones: el individualismo, el derecho de crítica, la autonomía de acción y la propia filosofía idealista. Teniendo en cuenta este análisis, Habermas destaca que el mayor logro de la modernidad es la conciencia de la propia normatividad. Dicho en otras palabras, si la modernidad es una forma privilegiada de conciencia histórica, se debe a que la racionalidad humana se hace consciente de su propia normatividad. Habermas se entronca así con la línea kantiana que hace de la autonomía del deber el quicio sobre el que sustentar el edificio de la Modernidad.

No todos los autores son tan benévolos con la modernidad. Nos referimos a los pensadores que, aún dentro de la matriz europea, han sido capaces de realizar una autocrítica consistente, haciendo visibles las debilidades de la razón moderna. A los ya mencionados Adorno, Horkheimer, Vattimo y Lyotard añadimos otros nombres como Walter Benjamin, Hannah Arendt y Agnes Heller.

Walter Benjamin tuvo el mérito de desentrañar la dinámica interna del historicismo para hacer valer las voces de los vencidos de la modernidad. Haciendo una sorprendente interacción entre teología y marxismo, Benjamin interpreta el desarrollo interno del capitalismo como la asunción de una culpa cada vez mayor. Parte de la identificación del capitalismo con la culpa (teológicamente concebida) y, de esta forma, asigna a la historia del capitalismo en la época moderna una culpa que 
aumenta progresivamente. El capitalismo de la modernidad se funda, para Benjamin, en una distorsión de la representación de la historia como un proceso continuo, en el que se da la confusión de naturaleza e historia. El capitalismo, al naturalizar la explotación de los hombres, convierte el decurso histórico en la instancia legitimadora de tal explotación. Por ello, Benjamin se alza contra la idea del progreso nacida al albur de la modernidad, la cual no hace más que servir de coartada para la continua explotación de la humanidad:

La idea de un progreso del género humano en la historia es inseparable de la representación de su movimiento como un avanzar por un tiempo homogéneo y vacío. La crítica de esta representación del movimiento histórico debe constituir el fundamento de la crítica de la idea de progreso en general. (Benjamin, 2010, p. 28)

Ante esta homogeneidad del tiempo histórico, Benjamin propone la noción de tiempo disyuntivo y pleno. EL pensador berlinés se nutre del concepto teológico del tiempo, para indicar que, frente a la linealidad del tiempo del progreso moderno, es posible pensar el tiempo del instante, el tiempo cualitativo en el que es posible pensar la diferencia.

Esta heterogeneidad pensada por Benjamin es actualizada en las trayectorias de Arendt y Heller. Para Hannah Arendt, con la modernidad asistimos a la ruptura de la tradición. Nos hemos quedado huérfanos porque los conceptos con los que podríamos pensar la realidad política han quedado desvinculados de las experiencias de las que brotaron. Por ello, admite que nuestra época es la de las "conchas vacías" (1996, p. 21). Si hay un hecho que constata esta ruptura es el fenómeno totalitario, el cual certifica, por un lado, la marcha inexorable de la mecánica ideológica y, por otra, abre un abismo de comprensión ante la novedad de tal hecho. Arendt, a partir de las atrocidades nazis y del esquema ideológico que justificaba su proceder, se embarcó en una investigación sobre las raíces modernas de los totalitarismos (2006). Asume que, junto factores de variada procedencia - racismo, antisemitismo, imperialismo, etc.- hay un esquema de pensamiento que cobra carta de naturaleza en la lógica totalitaria: el pensamiento como cálculo de consecuencias, en el esquema medios-fin. Arendt, vincula, de esta manera la filosofía 
de Hobbes con los regímenes totalitarios del siglo XX advirtiendo una homogeneización en la forma de pensar.

Al igual que Benjamin con el capitalismo, Arendt asigna al totalitarismo la fusión de naturaleza e historia y al igual que él, valora la diferencia, lo heterogéneo y la pluralidad. No en vano, la filosofía de Arendt se transforma en política de la mano de la valoración del ámbito público y la necesaria pluralidad que confiere sentido a tal espacio de aparición. Así, lo político no es sino el desafío de lo homogéneo. Arendt encuentra en la pluralidad el fundamento ontológico para que los seres humanos puedan efectivamente constituirse como seres políticos.

Agnes Heller, critica con la deriva de la modernidad, asume, también, un paradigma pluralista. Para ella, la modernidad ha supuesto un movimiento de asimilación de la libertad por parte de las instituciones. La gran denuncia de Heller (1995) es que en la época moderna, la razón ha devorado la libertad. Por ello, aunque asume el movimiento de la dialéctica hegeliana, adopta una postura crítica con el idealismo de la modernidad, vinculando la libertad y la vida en lo que ella llama una "dialéctica real" (por oposición a la ideal). Descubrimos dos fases en su pensamiento. Una primera, en que postula que la tarea de la filosofía consiste en la discusión valorativa. Es por ello que habla de una "filosofía radical", en la que el ejercicio racional se centra en la interpretación de los valores. En un segundo momento transita hacia una filosofía pluralista en la que la normatividad de un ethos compartido pueda tener fuentes metafísicas dispares:

Podría aun aceptar unas cuantas normas (prácticas) y valores como obligatorios, sobre todo unas normas morales y unos valores cuyo carácter obligatorio proceda de una diversidad de metafísicas, visiones del mundo y valores generales. (Heller, 1989, p. 34)

Las apreciaciones de Benjamin, Arendt o Heller nos hacen advertir que la discusión en torno a la modernidad y su interpretación no ha desaparecido. Si hemos sugerido brevemente su pensamiento, es para indicar que las sociedades postseculares postuladas por Habermas, pueden ser pensadas y vividas desde paradigmas metafísicos pluralistas. Con ello 
abrimos la puerta a que una filosofía pluralista e intercultural pueda presentarse como condición trascendental de la traducción intercultural.

\section{Interculturalidad como horizonte. La nueva condición trascendental}

La tesis doctoral de Daniel Berisso (2014) ha trazado líneas que nos permiten entender la tarea intercultural desde el concepto de ciudadanía, vinculando la ética intercultural y la ética de la liberación. Considerar la memoria de las víctimas (y en muchas ocasiones hablamos de víctimas culturales) nos lleva a hablar de la interculturalidad como un ethos liberador. Fue esta la intuición fundamental de Raúl FornetBetancourt (2004) al analizar el caso latinoamericano: la interculturalidad es una asignatura pendiente porque nace de la demanda de justicia cultural. Por ello, el autor cubano evidencia nuestro "analfabetismo intercultural" (2001) dado que, en nuestras sociedades, aún queda un largo camino para reparar las injusticias del pasado y para implementar definitivamente un ethos liberador. Resulta revelador el hecho de que la interculturalidad no pueda nombrarse formalmente, sino más bien intuirse tentativamente. Fornet-Betancourt coincide con Wimmer en el centrismo "tentativo". No tanto en el concepto de centro, cuanto en la indicación probatoria de lo que sea la interculturalidad. La interculturalidad nos obliga a redimensionar el punto de apoyo. El discurso intercultural nos involucra como protagonistas, de tal manera que las propias referencias de la identidad se orientan al encuentro con lo/ los otro/s. Siendo así, descubrimos que las identidades son, en realidad, relaciones abiertas, identidades compartidas. Por ello, si hablamos de "perspectiva" intercultural no podremos ya tratar de delimitar los espacios, sino de esbozar un horizonte.

El horizonte intercultural nos remite, a mi juicio, a dos fuentes básicas. En primer lugar, a la hermenéutica de Gadamer y, en segundo lugar, al conocimiento trascendental de Kant. Gadamer había tratado de clarificar la hermenéutica a través del establecimiento de la conocida tesis de la "fusión de horizontes". Un horizonte para Gadamer es "el ámbito de visión que abarca y encierra todo lo que es visible desde un determinado punto" (1998a, p. 372). Gadamer presenta la tarea com- 
prensiva como una fusión de horizontes (Horizontverschmelzung): el horizonte del intérprete y el horizonte del texto se funden haciendo de la comprensión una tarea infinita. Esto es posible porque la noción de horizonte es lo suficientemente flexible y dinámica como para ofrecer la transformación: "el horizonte de significados u horizonte de la historia efectual, es lo que vamos haciendo en nuestro camino y el cual el camino irá haciendo con nosotros. Por lo tanto, el horizonte de significado no es fijo, sino que se encuentra en perpetuo movimiento" (Gadamer 1998a, p. 337). Desde estos presupuestos hermenéuticos, comprendemos que el horizonte intercultural demande una rectificación constante del punto de partida. La propia identidad no es nunca un receptáculo cerrado. La filosofía de Gadamer se alinea así con las intuiciones básicas de Nussbaum y Fornet-Betancourt.

La segunda fuente de la noción de horizonte podemos encontrarla en Kant. Para Kant, existen unas condiciones empíricas del conocimiento y unas condiciones trascendentales. Las primeras hacen relación al contenido, objeto o materialidad del conocimiento, mientras que lo trascendental indica lo formal o a-priori del conocimiento. Si la experiencia sensible nos ofrece los datos materiales de conocimiento, nuestras propias estructuras cognitivas ofrecen las condiciones para que esos datos sean ordenados y, de esta forma se genere el conocimiento. A esas condiciones de conocimiento llama Kant "trascendental". En la segunda edición de la Crítica de la Razón Pura indica lo siguiente: "llamo trascendental a todo conocimiento que se ocupa no tanto con objetos como con nuestro modo de conocimiento de objetos en general, en cuanto este debe ser a priori" (1998, B25). Así, en la Crítica de la Razón Pura, Kant trataba de establecer las condiciones de conocimiento centrado en la constitución del objeto. Lo trascendental no sobrepasa nuestra experiencia, sino que la precede y hace posible el conocimiento del objeto. Años más tarde, Kant presentó lo trascendental en la dirección de la subjetividad universal. En la Crítica del Juicio se pregunta por la posibilidad de que el juicio de gusto sea universal. Si en la Crítica de la Razón Pura el análisis del conocimiento basculaba sobre la existencia de objetos, en la Critica del Juicio la pregunta por lo bello le hace analizar las estructuras representativas del sujeto. A partir de una representación sin objeto (lo que Kant llama el libre juego de la 
imaginación y el entendimiento) lo bello queda referido universalmente por su desinterés y su comunicabilidad. Kant desliga, así lo estético de lo cognoscitivo. La mera observación de la forma, libera la imaginación para poder emitir un juicio de gusto:

En el juicio de la belleza libre (según la mera forma) el juicio de gusto es puro. No se presupone ningún concepto de algún fin, en orden al cual lo múltiple serviría al objeto, ni se presupone, pues, lo que éste debe representar. Presuponer eso no haría sino limitar la imaginación, que, por así decirlo, juega en la observación de la formal. (Kant, 1974, p. 80)

Teniendo en cuenta que lo trascendental funciona como una condición ineludible de conocimiento y que la imaginación es capaz de hacer de lo bello algo comunicable, considero posible establecer el "horizonte intercultural" como una nueva condición trascendental de la agenda política intercultural en las sociedades postseculares. Se trataría así de un requisito básico para que la interculturalidad pueda darse en toda su extensión dinámica. En mi opinión, entender que el horizonte intercultural funciona como una condición trascendental:

- Garantiza la pluralidad sin la reducción a la homogeneidad. La propuesta de Agnes Heller acerca de derivar propuestas normativas comunes de fuentes metafísicas plurales queda así garantizada.

- Permite avanzar de manera no coercitiva en la dirección normativa. A lo largo de este ensayo nos hemos referido en algunas ocasiones a una moral intercultural, la cual no podría realizarse sin un ethos liberador. Precisamente por este ethos de libertad no podía imponerse ningún programa intercultural en el que la folclorización impida, por una parte, una auténtica traducción cultural y pretenda, por otra, instaurar cualquier tipo de etnocentrismo.

- Implica asumir sistemas epistémicos más allá de su formulación formal. Un horizonte intercultural como condición trascendental impide pretender igualar las condiciones "lógicas" de presentación de los sistemas epistémicos. Un planteamiento verdaderamente intercultural nos autoriza a aceptar diversas expresiones de la racionalidad sin que se vean reducidas a su concentración conceptual. 
- Evita el contenido proposicional de las culturas despejando el camino para la traducción cultural. Una condición trascendental, en el sentido estético kantiano, elude cualquier dato empírico y se centra en las estructuras comunicables del ser humano. La relevancia estética que Kant otorga a la imaginación hace de esta un poderoso instrumento para que el ejercicio de traducción cultural cumpla con dos requisitos básicos: el desinterés y la comunicabilidad.

\section{Más allá de la traducción}

Volvamos por un momento a Habermas. Pensando en la implementación de una sociedad postsecular, Habermas concibe la realización completa de la agenda de la secularización en los siguientes términos: "una secularización no aniquiladora se realiza en el modo de la traducción" (Habermas, 2001a, p. 29). Habermas está pensando en la traducción de los particularismos culturales y religiosos a un lenguaje universal, fundado, según su opinión, en la razón moderna.

Desde mi punto de vista, se trata de una traducción que no permite entender en toda su amplitud y profundidad la traducción intercultural. Para entender dicha traducción intercultural estimo oportuno acudir brevemente a la lingüística. Jacokbson (1959) planteó tres posibles modos de entender la traducción: intralingüística, interlingüística y la transmutación. La traducción intralingüística corresponde a una interpretación de signos verbales por medio de otros signos del mismo lenguaje. La traducción interlingüística o "traducción propiamente dicha" es la interpretación de los signos verbales por medio de otro lenguaje. Transmutación es un movimiento inter-semiótico: "La traducción o transmutación intersemiótica es una interpretación de los signos verbales mediante signos de sistemas de signos no verbales" (Jakobson, 1959, p. 233).

Recientemente Carlo Severi ha ido más allá de Jakobson. Teniendo en cuenta la relación entre el grafismo amerindio y las facultades implicadas en el conocimiento ha propuesto una "transmutación propiamente dicha”. Severi se apoya en la recepción sensorial que las culturas amazónicas hacen de los diseños de ciertos objetos. Los sistemas no verbales son "traducidos" a otro tipo de sistemas (acústicos, verbales, vi- 
suals, etc.) Severi puede afirmar que: "traducción" (que involucra tareas cognitivas específicas) opera no solo entre diferentes culturas (o idiomas), sino también entre diferentes contextos pragmáticos en el mismo idioma y entre formas de expresión lingüísticas y no lingüísticas, incluso dentro de sociedades individuales" (2014, p. 45).

La transmutación a la que nos lleva Severi existe, por lo tanto, entre vías de expresión lingüísticas y no lingüísticas, tanto en sociedades que presentan un único sistema epistémico, como en aquellas sociedades en las que conviven varios sistemas epistémicos. La secularización, en este aspecto, no debería arrogarse pretensión alguna. Lo que Habermas está indicando es la necesidad de transmutación a un término lingüístico universalmente reconocido. Teniendo en cuenta la naturaleza pluralista de las sociedades postseculares, la cuestión no es tan sencilla.

Esta transmutación "propiamente dicha" de la que habla Severi, apunta en la misma dirección que la antropología del arte de Alfred Gell (1998) y el análisis comparativo que Lloyd (2014) realiza entre los sistemas griego y chino. Gell se vio obligado a hablar de la indexicalidad del arte para referir la agencia social de los artefactos artísticos. Un índice es para Gell: "una entidad material que provoca inferencias abductivas, interpretaciones cognitivas, etc." (1998, p. 27). Para Gell, la traducción de lo indicado en el arte a otros sistemas no implica necesariamente operaciones deductivas ni translaciones directas de sentido. Gell, apoyándose en Pierce y en Umberto Eco, acude a la abducción como aquella operación donde hacemos suposiciones tentativas acerca del sentido de unos signos de los que desconocemos su significado.

Creo oportuno reconocer que esta operación bien podría ser un camino de traducción intercultural y que en la vida diaria es realizado por numerosos pueblos. Se trata de un mecanismo que aúna los elementos en los que hemos insistido en este ensayo. Por una parte, reconoce el carácter tentativo y abierto del acercamiento entre culturas (como indicaba Wimmer en su centrismo tentativo).

Por otra parte, se apoya en la misma facultad kantiana reservada para cuestiones estéticas: la imaginación. La abducción descubre que en toda inferencia semiótica, y la traducción intercultural lo es, descubrimos zonas de conjetura difíciles de convertir en otro sistema epistémico: 
La abducción cubre el área gris donde la inferencia semiótica (de los significados de los signos) se fusiona con inferencias hipotéticas de un tipo no semiótico (o no convencionalmente semiótico) como la inferencia de Kepler a partir del movimiento aparente de Marte en el cielo nocturno, en el que el planeta describía una trayectoria elíptica. (Gell, 1998, p. 14).

Geoffrey Lloyd ha comparado los sistemas epistémicos chino y griego en torno al debate sobre la constitución de la realidad. Vinculando el debate en torno a lo que podríamos llamar la filosofía de la naturaleza, admite que necesitamos pensar en términos de "elasticidad semántica" (Lloyd, 2014, p. 73) para darnos cuenta de que la referencialidad lingüística va de la mano de sistemas epistémicos. Así, Gadamer se queda corto cuando presenta la fusión de horizontes en términos lingüísticos. Cuando en la última parte de Verdad y método presenta el problema del "mundo en sí" y la posibilidad de referenciarlo mediante una común lingüisticidad está incurriendo en el mismo logocentrismo que hemos visto en Habermas.

Pensar una traducción intercultural en términos de transmutación (Severi) o abducción (Gell) nos hace considerar un concepto expandido, dinámico y cambiante de cultura. Agentes de traducción serán así los elementos lingüísticos, pero también los índices artísticos, las concepciones estéticas, las acciones normativas, las relaciones en torno a la gastronomía, etc. Desde aquí no sería posible pensar una sociedad postsecular desde el punto de vista de la mera reducción a contenidos racionales normativos. En nuestras sociedades postseculares, plurales y desiguales, la agenda intercultural se revela como una tarea ineludible, en la que el carácter normativo viene dado por las posibilidades de transmutación entre sistemas epistémicos.

\section{Bibliografía}

Adorno, Th., \& Horkheimer, M. (2007). Dialéctica de la Ilustración. Madrid: Akal.

Altmann, Ph. (2013). Plurinacionality and Interculturality in Ecuador: The Indigenous movement and the Development of Political Concepts. Ibe- 
roamericana. Nordic Journal of Latin American and Caribbean Studies, 1(XLIII), 47-66.

(2017). La interculturalidad como concepto politico y one fits all: Un acercamiento a un punto nodal del discurso político ecuatoriano. En J. Gómez Rendón (Ed.), Repensar la interculturalidad (pp. 13-36). Guayaquil: UArtes Ediciones.

Arendt, H. (1996). Entre el pasado y el futuro. Barcelona: Península. (2006). Los orígenes del totalitarismo. Madrid: Alianza.

Asad, T. (2003). Formations of the secular: Christianity, Islam, Modernity. Stanford: Stanford University Press.

Barry, B. (2001). Culture and Equality. An Egalitarian Critique of Multiculturalism. Cambridge: Polity Press.

Bauman, Z. (2010). Modernidad y holocausto. Madrid: Sequitur.

Benjamin, W. (2010). Tesis sobre la historia y otros fragmentos [ed. y trad. de Bolívar Echeverría]. Bogotá: Desde abajo.

Berisso, D. (2014). Los limites del concepto de ciudadanía en el marco de una ética Latinoamericana: de una ética de la liberación de la praxis intercultural. Buenos Aires: Editorial de la Facultad de Filosofía y Letras Universidad de Buenos Aires.

Bhabha, H. (1994). El lugar de la cultura. Buenos Aires: Manantial.

Carbonell i Cortés, O. (1997). Traducir al Otro: Traducción, exotismo, post-colonialismo. Cuenca: Ediciones de la Universidad de Castilla-La Mancha.

Casanova, J. (2006). Rethinking secularization. The Hedgehog Review, 8(1-2), 7-22.

Chakrabarty, D. (2011). The Muddle of Modernity. The American Historical Review, 116(3), 663-675. https://doi.org/10.1086/ahr.116.3.663

Derrida, J. (1986). De la Gramatología. México: Siglo XXI.

Estermann, J. (2012). Apu Taytayku: Religion und Theologie im andinen Kontext Lateinamerikas (Theologie Interkulturell) Ostfildern: Grünewald.

Fornet-Betancour, R. (2001). Lo intercultural: el problema de su definición. Encuentro Internacional sobre interculturalidad. Barcelona: CIDOB. (2004). Crítica intercultural de la filosofía latinoamericana actual. Madrid: Trotta.

Gadamer, H. G. (1998a). Verdad y Método I. Salamanca: Sígueme. 
Gauchet, M. (2005). El desencantamiento del mundo. Una historia politica de la religión. Madrid: Trotta.

Gell, A. (1998). Art and Agency: an anthropological Theory. Oxford: Oxford University Press.

Gómez, R. (2014). La dimensión valorativa de las ciencias. Hacia una filosofía politica. Bernal: Universidad Nacional de Quilmes.

Gómez Rendón, J. (2017). Aproximaciones semióticas a la interculturalidad. En J. Gómez Rendón (Ed.), Repensar la interculturalidad (pp. 109-157). Guayaquil: UArtes Ediciones.

Habermas, J. (1981). Theorie des kommunikativen Handelns. Frankfurt: Suhrkamp

(1988). Nachmetaphysisches Denken. Frankfurt: Suhrkamp.

(1989). El discurso filosófico de la modernidad (Doce lecciones). Madrid: Taurus.

(2001a). Glauben und Wissen. Frankfurt: Suhrkamp.

(2001b). Die Zukunft der menschlichen Natur. Auf dem Weg zu einer liberalen Eugenik? Frankfurt: Suhrkamp

(2007). Ein Bewusstsein von dem, was fehlt. Über Glauben und Wissen und den Defaitismus der modernen Vernunft. Neue Zürcher Zeitung, 10 de febrero.

Heller, A. (1989). La situación moral en la modernidad. En Políticas de la postmodernidad. Ensayos de crítica cultural. Barcelona: Península.

(1995) El péndulo de la modernidad. Barcelona: Península.

Hume, D. (1984). [1739-1740] Tratado de la naturaleza humana, tomo 3. Buenos Aires: Hyspamerica.

Huntington, S. (1996). The Clash of Civilizations and the Remaking of World Order. New York: Simon \& Schuster.

Jakobson, R. (1959). On linguistic aspects of translation. On Translation, 3, 232-239.

Jaspers, K. (1949). Vom Ursprung und Ziel der Geschichte. Munich: Piper.

Joas, H. (2002). Eine Rose im Kreuz der Vernunft. Die Zeit, 7 de febrero.

Kant, I. (1998) [1787, 1781] Kritik der reinen Vernunft. Hamburgo: J. Tim- mermann y H. Klemme ed. 
(1974) [1790] Kritik der Urteilskraft. Hamburgo: Meiner.

Klages, L. (1972). [1929-32] Der Geist als Widersacher der Seele Hauptwerk. Bonn: Bouvier.

Kymlicka, W. (1996). Ciudadanía multicultural. Una teoría liberal de los derechos de las minorías. Barcelona: Paidós.

Lloyd, G. (2014). Being, Humanity and Understanding. Oxford: Oxford University Press.

Loewe, D. (2012). La utopía multicultural. Rev. Inter. Mob. Hum., XX(38), 4565. Brasilia.

Lyotard, F. (1987). La condición postmoderna. Informe sobre el saber. Madrid: Cátedra.

Mardones, J. M. (1998). El discurso religioso de la modernidad. Habermas y la religión. Barcelona: Anthropos.

Martin, D. (1978). A General Theory of Secularization. New York: Harper \& Row.

Nussbaum, M. (1997). Cultivating Humanity. Cambridge: Harvard University Press.

Parekh, B. (2006). Rethinking Multiculturalism. Cultural Diversity and Political Theory. Hamshire, UK.: Palgrave Macmillan.

Rodríguez Duplá, L. (2017). Sobre los dos sentidos del concepto habermasiano de sociedad postsecular. Daimon. Revista Internacional de Filosofía, 70, 23-39. http://dx.doi.org/10.6018/daimon/223291

Said, E. (1978). Orientalism. Nueva York: Vintage Books.

Searle, J. (1964). How to Derive 'Ought' from 'Is'. Philosophical Review, 73, 43 58.

(1997). La construcción de la realidad social. Barcelona: Paidós.

Severi, C. (2015). (éd. avec William Hanks). Translating Worlds: The Epistemological Space of Translation. Chicago: University of Chicago Press, HAU Books.

(2014). Trasmutating beings. A proposal for an anthropology of thought. Hau: Journal of Ethnographic Theory, 4(2), 41-71. http:// dx.doi.org/10.14318/hau4.2.003

Sloterdijk, P. (1999). Normas para el parque humano. Madrid: Siruela.

Spivak, G.Ch. (1987). In Other Worlds: Essays in Cultural Politics. New York: Routledge. 
Taylor, Ch. (2007). A Secular Age. Cambridge, MA: Harvard University Press. (1994). The Politics of Recognition. En A. Gutmann (Ed.), Multiculturalism. Princeton: Princeton University Press.

Tully, J. (1995). Strange Multiplicity. Constitutionalism in an Age of Diversity. Cambridge: Cambridge University Press.

Vattimo, G. (1983). Il pensiero debole. Milán: Feltrinelli. (1985). La fine della modernità. Milán: Garzanti.

Viveiros de Castro, E. (1998). Cosmological deixis and Amerindian perspectivism. Journal of the Royal Anthropological Institute (N.S.), 4, 469-88.

Vygotsky, L. S. (1978). Mind in society. Cambridge MA: Harvard University Press.

Waldmüller, J. (2017). Aportes desde la filosofía intercultural al debate ecuatoriano: un acercamiento a partir de la teoría de los centrismos. En J. Gómez Rendón (Ed.), Repensar la interculturalidad (pp. 72-108). Guayaquil: UArtes Ediciones.

Wimmer, F. M. (2004). Einfuhrung in der Interkulturelle Philosophie. Viena: UTB Verlag.

Young, I. M. (1990). Justice and the Politics of Difference. Princeton: Princeton University Press. 